\title{
Grenzen des Vorsorgestaats in einer alternden Gesellschaft
}

\author{
Jochen Pimpertz
}

Online publiziert: 24. November 2020

(C) Der/die Autor(en) 2020

\section{Die ökonomische Perspektive}

Der Begriff Vorsorgestaat setzt Risikovorsorge und staatliches Handeln in einen unmittelbaren Zusammenhang. Dies mag beobachtbaren Praktiken der Verantwortung entsprechen (Ewald 1993), gleichwohl ist dieser Terminus der Ökonomie fremd. Denn mehr noch als die Beobachtung eines zunehmenden staatlichen Gestaltungsanspruchs sind die Fragen, für welche Risiken Vorsorge zu treffen ist, ob und unter welchen Zielsetzungen dies vorteilhaft in individueller oder kollektiver Verantwortlichkeit geschieht und welche Rolle dabei dem Staat zukommt, Gegenstand wirtschaftswissenschaftlicher Forschung.

Dabei geht es um das Abwägen der wirtschaftlichen Folgen, die einerseits bei einer marktnahen, eigenverantwortlichen Lösung unter Wahrung individueller Handlungsfreiheiten zu erwarten sind, andererseits bei einer marktferneren Lösung unter staatlicher Einschränkung ebendieser Freiheiten (Watrin 1984). Da diese Abwägung auf einem der Disziplin eigenen liberalen Werturteil basiert (zum methodologischen Individualismus Roth 2014, S. 1 ff.), stehen ökonomische Argumente regelmäßig im Konflikt mit außer-ökonomisch begründeten Normen, die zur Rechtfertigung des staatlichen Gestaltungsanspruchs angeführt werden. In einem interdisziplinären Forum sind deshalb ordnungsökonomische Beiträge als normativ zu kennzeichnen.

Die Rolle des Vorsorgestaats kann aber auch mit Blick auf die Anreize, Handlungsmöglichkeiten und Restriktionen politischer Akteure diskutiert werden (Roth 2014, S. $217 \mathrm{ff}$.). Im Folgenden wird dazu argumentiert, dass sich mit dem demografischen Wandel intergenerative Verteilungskonflikte verschärfen. Dabei steht die These im Raum, dass sich demokratisch legitimierte Agenten angesichts ei-

Dr. rer. pol. J. Pimpertz $(\bowtie)$

Öffentliche Finanzen, Soziale Sicherung, Verteilung, Institut der deutschen Wirtschaft, 101942, 50459 Köln, Deutschland

E-Mail: pimpertz@iwkoeln.de 
nes steigenden Alters des Medianwählers eher an den Versorgungsinteressen älterer Wählergruppen orientieren werden - dies ließe eine Ausweitung des staatlichen Gestaltungsanspruchs erwarten. Andererseits werden aber die Opportunitätskosten großzügiger Leistungsversprechen deutlicher spürbar als bislang. Beschreibt der Begriff Vorsorgestaat also eine zunehmende Politisierung der Begriffe Risiko und Vorsorge, dann wird hier die Gegenthese begründet, dass der damit einhergehende Gestaltungsanspruch für demokratisch legitimierte Akteure selbst zu einem politischen Risiko wird.

\section{Sozialversicherungen vor demografischen Herausforderungen}

Im Folgenden werden dazu die Systeme der gesetzlichen Renten-, Kranken- und Pflegeversicherung in den Blick genommen. Über sie werden weite Teile der Bevölkerung verpflichtet, Vorsorge im Sinne einer Absicherung gegen zentrale Lebensrisiken zu betreiben. Systemprägend ist deren Finanzierung im Umlageverfahren, wonach die jährlich auftretenden Ausgaben aus dem aktuellen Aufkommen der lohn- und rentenbezogenen Beiträge finanziert werden, gegebenenfalls um Steuerzuschüsse ergänzt. Auch das Solidaritätsprinzip wird als systemprägend beschrieben, mit dem Abweichungen von privatwirtschaftlichen Versicherungslösungen begründet werden.

Für die Risiken Alterseinkommen, Krankheit und Pflegebedürftigkeit ist typisch, dass die Versicherungsschäden regelmäßig im Alter auftreten bzw. deren Wahrscheinlichkeit und Höhe mit dem Alter steigen. In der Gesetzlichen Rentenversicherung (GRV) ist dies unmittelbar durch den Wechsel von der beitragspflichtigen Erwerbsphase in den Rentenbezug gegeben. Aber auch in der Gesetzlichen Krankenversicherung (GKV) und noch ausgeprägter in der Sozialen Pflegeversicherung (SPV) steigen die durchschnittlichen Ausgaben altersabhängig (Kochskämper 2017, S. 5 f.).

Mit der Alterung der geburtenstarken Jahrgänge wechseln ab Mitte dieses Jahrzehnts vermehrt Menschen in den Ruhestand, während aufgrund niedriger Geburtenraten weniger junge Menschen in das Erwerbsleben folgen. In der GRV steigt deshalb die Zahl der Rentenbezieher bis zum Jahr 2040 von derzeit knapp 20 auf über 24 Mio. und verharrt danach auf mindestens diesem Niveau, während die Zahl der Beitragszahler bis zum Jahr 2040 von rund 35 auf dann gut 32 Mio. und weiter bis auf rund 30 Mio. im Jahr 2060 schrumpfen wird (Basisszenario der Kommission Verlässlicher Generationenvertrag 2020, S. 55). In der GKV und SPV tragen Ruheständler zwar weiterhin zur Finanzierung bei. Aber im Durchschnitt fällt das beitragspflichtige Alterseinkommen niedriger aus als das entsprechende Erwerbseinkommen. Steigt künftig der Anteil der Ruheständler mit unterdurchschnittlichem Beitrag, dann werden damit gleichzeitig die Altersklassen mit überdurchschnittlich hohen Leistungsausgaben häufiger besetzt (Kochskämper und Pimpertz 2015, S. 111).

Daraus erwächst eine implizite Schuld, die das Ausmaß der nicht nachhaltig finanzierten Leistungsversprechen in den sozialen Sicherungssystemen beschreibt. Sie errechnet sich annahmebasiert aus der Simulation der künftigen Einnahme- und 
Ausgabeströme aller Kohorten bei gegebenem Leistungs- und Beitragsrecht. So summierte sich im Jahr 2018 der Barwert der bei unveränderten Beitragssätzen künftig nicht über das Beitragsaufkommen zu finanzierenden Leistungsversprechen nach Bahnsen et al. (2018, S. 12) für GRV, GKV und SPV zusammen auf das 2,7-Fache des Bruttoinlandsprodukts.

Diese implizite Schuld wird explizit, sobald die notwendigen Beitragssatzanpassungen unter der Annahme simuliert werden, dass die Leistungsversprechen für alle künftig Anspruchsberechtigten erfüllt werden. Errechneten Aretz et al. (2016) einen Anstieg der Beitragssatzsumme (einschließlich Arbeitslosenversicherung) von derzeit knapp 40 auf 45,5\% im Jahr 2040, so lag der Wert bei Ehrentraut et al. (2017) je nach unterstellter Wachstumsdynamik der Gesundheitsausgaben zwischen 48,8 und $55,8 \%$. Werding und Läpple (2019) kommen in ihrem Referenzszenario auf 49,6\%.

\section{Verteilungskonflikte in einer alternden Bevölkerung}

Dieser Anstieg der Beitragssätze verdeutlich die intergenerative Lastverschiebung: Auch wenn im Umlageverfahren jederzeit ein Ausgleich zwischen Jung und Alt realisiert werden kann, müssen in einer alternden Versichertengemeinschaft die Mitglieder der jeweils jüngeren Kohorten über den gesamten Lebenszyklus höhere Beitragslasten schultern als die Mitglieder älterer Kohorten - ohne dafür durch höhere Leistungen kompensiert zu werden (für die GKV Pimpertz 2018, S. 56ff.). Damit wird eine politische Abwägung zwischen den Interessen der rentennahen Jahrgänge und Ruheständler einerseits und denen der beruflich aktiven, jüngeren Jahrgängen andererseits unausweichlich.

Geht man nun davon aus, dass sich politische Agenten aufgrund ihrer Wiederwahlinteressen an den vermeintlich mehrheitlich geteilten Motiven der Wähler orientieren und dass sich gleichzeitig der Medianwähler in höhere Altersklassen verschiebt, dann legt dieses Kalkül eine Ausrichtung politischer Programmatik an den Versorgungsinteressen einer älter werdenden Wählerschaft nahe (grundlegend Downs 1968). Zu erwarten wäre demnach eine wachsende Bedeutung des Vorsorgestaats.

Diese Überlegung lässt sich am Beispiel der Rentenpolitik plausibilisieren. Denn war die Reformpolitik im vorvergangenen Jahrzehnt noch durch einen Paradigmenwechsel geprägt, wonach die GRV von ihrem ursprünglichen Versprechen einer den Lebensstandard sichernden Versorgung abgerückt ist, so wurden in den letzten Jahren die Wirkungen dieser Reformen in Teilen korrigiert - zum Beispiel durch die privilegierte Behandlung besonders langjährig Versicherter bei vorzeitigem Rentenbezug (abschlagsfreie Rente mit 63), die Festschreibung des Mindestsicherungsniveaus auf 48 statt $43 \%$ bis zum Jahr 2025 (Rentenpakt) oder die steuerfinanzierte Aufwertung niedriger Anwartschaften unter bestimmten Voraussetzungen (Grundrente). Bislang ließ sich das zur Finanzierung notwendige Beitragsaufkommen aufgrund der günstigen Beschäftigungsentwicklung mit einem sinkenden bzw. niedrigen Beitragssatz generieren. Aber bereits in den kommenden Jahren droht ein steiler Anstieg allein des Beitragssatzes zur GRV - mit wirtschaftlichen und verteilungspolitischen Folgen. 
Denn bei gegebener Produktivitätsentwicklung und gleichermaßen wachsenden Bruttolöhnen führen höhere Beitragssätze nicht nur dazu, dass das Nettolohnwachstum hinter der Entwicklung der Produktivität zurückbleibt, weil ein immer größerer Anteil des erwirtschafteten Verteilungsspielraums für die Finanzierung der Sicherungsansprüche vorrangig älterer Versicherter vereinnahmt wird (intergenerativer Verteilungskonflikt). Auch die Arbeitskosten der Unternehmen steigen unter der Annahme einer produktivitätsorientierten Bruttolohnentwicklung. Denn die Arbeitgeberbeiträge werden auf der Grundlage des Bruttolohns berechnet, deshalb steigen die Arbeitskosten bei wachsenden Beitragslasten stärker als die Produktivität - verbunden mit negativen Beschäftigungsanreizen (tarifpolitischer Verteilungskonflikt). Gelingt es der Arbeitnehmervertretung, konstante Nettolöhne durchzusetzen, steigen die Arbeitskosten noch deutlicher und beschäftigungskritische Anreize fallen stärker ins Gewicht.

Damit verschärft der grundlegende intergenerative Verteilungskonflikt mittelbar auch den tarifpolitischen Verteilungskonflikt. Die Finanzierungslast großzügiger Leistungsversprechen lässt sich mit der Alterung der geburtenstarken Jahrgänge nicht länger in eine ferne Zukunft verschieben, die ökonomischen Konsequenzen werden für Versicherte (und Wähler) zeitnah und zunehmend deutlich spürbar.

Das erschwert das Kalkül rational handelnder politischer Agenten. Denn mit einer Verschärfung des Knappheits- und Verteilungsproblems wird immer deutlicher, dass der staatliche Gestaltungsanspruch in Vorsorgefragen nicht nur die Versorgungsinteressen der rentennahen Jahrgänge tangiert, sondern auch die längerfristigen Einkommensperspektiven der jüngeren Kohorten. Das gilt insbesondere für die GRV, weil sich hohe Beitragslasten sowohl unmittelbar auf die Beschäftigungschancen jüngerer Beitragszahler auswirken können als auch mittelbar auf deren längerfristigen Einkommens- und Versorgungsperspektiven, da ihre künftigen Rentenansprüche beitragsbezogen ermittelt werden.

Für politische Akteure wird es noch schwieriger, die Interessen potenzieller Wähler einzuschätzen, wenn man angesichts einer wachsenden Konzentration der Kindererziehung auf weniger Familien unterstellt, dass die Motivation der Wähler auch davon beeinflusst wird, ob die Folgen der intergenerativen Lastverschiebung im Mehr-Generationen-Kontext bei den eigenen Kindern und Enkeln erlebt werden.

\section{Staatliche Handlungsoptionen und Restriktionen}

Wenn allein das Alter des Medianwählers den an ihrer (Wieder-)Wahl interessierten politischen Agenten kaum noch Orientierung bietet, dann stehen diese vor dem ungelösten Problem, den politischen Gestaltungsanspruch in Vorsorgefragen glaubhaft erfüllen zu können. Welche Handlungsalternativen stehen dem Vorsorgestaat zur Verfügung? Dazu sollen im Folgenden einige Optionen exemplarisch diskutiert werden. 


\subsection{Höhere Steuerzuschüsse}

Zunächst könnten (höhere) Steuerzuschüsse an die Sozialversicherungen den Anpassungsdruck mildern. Werden zum Beispiel in der GRV beitragsbezogen ermittelte Rentenansprüche zusätzlich aus Steuermitteln finanziert, lassen sich auf den ersten Blick moderate Beitragssatzanpassungen mit umfassenden Leistungsversprechen vereinbaren. Politische Agenten könnten sozialpolitische Handlungskompetenz signalisieren, die Rolle des Vorsorgestaats würde sichtbar gestärkt. Jedoch führt ein stärkeres finanzielles Engagement des Staats zurück auf das grundlegende Knappheitsproblem. Denn mit einem höheren Steuerzuschuss an die GRV (oder analog an die GKV und/oder die SPV) werden bei gegebenem Steueraufkommen politische Handlungsspielräume für konkurrierende Ziele eingeengt. Insbesondere wenn dies auf Kosten wirtschaftspolitisch begründeter Ausgaben realisiert würde, drohen volkswirtschaftliche Wachstumsperspektiven aus dem Blick zu geraten, die bedeutsam sind für das Generieren des Beitrags- und Steueraufkommens. Dieser Gedanke gilt analog, wenn eine Erhöhung des Steueraufkommens in Betracht gezogen würde, da höhere Grenzbelastungen mit negativen Leistungsanreizen einhergehen und die volkswirtschaftlichen Wachstumsperspektiven im internationalen Standortwettbewerb beeinträchtigen. Auch eine dauerhaft höhere Staatsverschuldung bietet keinen Ausweg. Zum einen bliebe die Notwendigkeit bestehen, den Steuerzuschuss unter dem Ziel der Beitragssatzstabilisierung stetig zu erhöhen. Zum anderen drohen - auch aufgrund einer steigenden Schuldenquote - Zinsänderungsrisiken, die die Kosten des Schuldendienstes erhöhen und den politischen Gestaltungsspielraum wiederum einengen.

\subsection{Exkurs: Rolle der sozialen Selbstverwaltung}

Die Rolle der sozialen Selbstverwaltung kann hierbei nur in einer Randnotiz diskutiert werden. Im internationalen Vergleich sticht der deutsche Sozialstaat durch die Organisation der Sozialversicherungssysteme hervor, die nicht unmittelbar der exekutiven Steuerung des Staats unterliegen, sondern typischerweise durch selbständige Gremien mit paritätischer Besetzung der Arbeitnehmer- und Arbeitgebervertretungen verwaltet werden. Diese Besonderheit folgt der ursprünglichen Idee der Sozialen Marktwirtschaft, die Müller-Armack (1950) als soziale Irenik beschreibt. Demnach lässt sich die politische und ökonomische Rolle sozialer Selbstverwaltung wie folgt interpretieren:

Die paritätische Besetzung der Gremien zwingt Arbeitgeber- wie Arbeitnehmervertretungen dazu, sich mit den Folgen ihrer tarifpolitischen Verhandlungen auch im Kontext sozialer Sicherung auseinanderzusetzen und somit mittelbar die gesellschaftlichen Konsequenzen in den Blick zu nehmen, die aus Tarifabschlüssen resultieren können. Beispielhaft und vereinfacht gesprochen erodiert mit dauerhaft niedrigen Lohnabschlüssen die Finanzierungsbasis der GKV, mit Folgen für Umfang und Qualität der gesundheitlichen Versorgung sowie mittelbar für die Produktivität der Arbeitnehmer in den Betrieben. Umgekehrt droht mit dauerhaft über der Produktivität liegenden Tarifabschlüssen Unterbeschäftigung, die sich unmittelbar auf die 
Finanzierung der Arbeitslosenversicherung, längerfristig aber auch auf die Alterssicherung der zu diesem Lohnniveau nicht zu beschäftigenden Personen auswirkt.

Das System - so die vereinfachende Interpretation - trägt damit dem Gedanken der Interdependenz der Ordnungen (Eucken 1950) in besonderem Maße Rechnung, weil es die Sozialpartner zwingt, über ihre unmittelbaren Eigeninteressen hinaus gesellschaftliche Konflikte zu befrieden. Als Forschungshypothese ließe sich formulieren, dass damit ein systematisch friedliebenderes (Streik-)Verhalten der Sozialpartner befördert wird, als es zum Beispiel beim französischen Nachbarn beobachtet werden kann. Folgt man dieser Hypothese, dann würde aber die Bedeutung eben dieser Sozialpartnerschaft mit einem steigenden Finanzierungsanteil aus Steuermitteln sukzessive ausgehöhlt und damit ein stabilisierendes Element der politischen Ordnung geschwächt.

\subsection{Erweiterung der Beitragsbemessung}

Vor allem für die GKV und SPV wird eine Erweiterung der Beitragsbemessung diskutiert. Das Einbeziehen bislang beitragsfreier Entgeltbestandteile verspricht ein niedrigeres Beitragssatzniveau, da ein unverändert hohes Beitragsaufkommen aus einer breiteren Bemessungsgrundlage generiert werden könnte. Dieser Effekt wäre aber ein einmaliger, weil die demografisch bedingte Ausgabendynamik davon unberührt bliebe. Für die Unternehmen ergäbe sich zudem keine Arbeitskostenentlastung, weil das Beitragsaufkommen lediglich anders auf die Entgeltgruppen verteilt würde.

Anders scheint die Beurteilung, wenn zusätzliche Einkommensquellen in die Beitragsbemessung einbezogen werden. Allerdings sind dann Ausweichreaktionen in den Blick zu nehmen. So käme zum Beispiel eine Beitragspflicht für Kapitaleinkommen einer Steuer gleich, die das Investitionskalkül verzerren würde - mit kaum vorhersehbaren Folgen für die volkswirtschaftlichen Entwicklungsperspektiven, weil sowohl mit Preisreaktionen im Inland zu rechnen wäre als auch mit einem Kapitalabfluss. Außerdem stellt sich die Frage, wie mit negativen Einkünften zum Beispiel aus Vermietung und Verpachtung beitragssystematisch zu verfahren wäre. Würde man analog zur Feststellung des steuerpflichtigen Einkommens verfahren, drohte in diesen Fällen die Bemessungsgrundlage verkürzt statt erweitert zu werden.

\subsection{Ausweitung der Pflichtversicherung}

Daneben wird eine Ausweitung des pflichtversicherten Personenkreises diskutiert in der Hoffnung, die Solidargemeinschaft der gesetzlich Versicherten stärken zu können. Diese Hoffnung beruht auf der Vermutung, dass bislang nicht gesetzlich Versicherte im Durchschnitt ein im Vergleich zum bestehenden Kollektiv höheres beitragspflichtiges Einkommen erzielen. Selbst wenn dies der Fall wäre, sind mögliche Folgen auf der Ausgabenseite bislang empirisch ungeklärt. Zum einen steht zu vermuten, dass mit den Beamten eine relativ alte Gruppe integriert würde, die unter dem Dach von GKV und SPV überdurchschnittlich hohe Ausgaben verursachen würde - die erhoffte Entlastung würde zumindest teilweise kompensiert. Zum anderen bliebe der längerfristige, demografisch bedingte Anpassungsdruck auf die Beitragssätze davon unberührt (Pimpertz 2019). 


\subsection{Obligatorium Privatvorsorge}

Alternativ ließe sich dem politischen Gestaltungsanspruch in Form einer verpflichtenden privaten und/oder betrieblichen Altersvorsorge nachkommen. Damit würde der Vorsorgestaat Vorkehrungen für den Risikoeintritt veranlassen, ohne selbst in die finanzielle Verantwortung treten zu müssen. Allerdings müssten dann zwingend auch Umfang und Höhe einer verpflichtenden Vorsorge definiert werden. Aus ökonomischer Sicht ist eine zwangsweise Absicherung über das Niveau der steuerfinanzierten Grundsicherung hinaus kaum zu begründen, insbesondere wenn im Rahmen der Sozialversicherungspflicht diese Schwelle im Regelfall überschritten wird. Mit einem Obligatorium drohen zudem individuell wie kollektiv rationale Entscheidungen zum Beispiel zugunsten beruflicher Weiterbildung verzerrt oder gar verhindert zu werden, da eine zwangsweise Verwendung knapper Ressourcen den Wirtschaftssubjekten Mittel für alternative Zwecke entzieht. Das gilt auch mit Blick auf Vorsorgealternativen, wenn durch ein Obligatorium die Wahl präferenzgerechter Vorsorgeformen (zum Beispiel Immobilienerwerb) eingeschränkt wird (Beznoska und Pimpertz 2016, S. 16f.).

\section{Politisierung von Vorsorge wird zum politischen Risiko}

Der demografische Wandel verschärft den intergenerativen Verteilungskonflikt, der sich aufgrund der lohnbezogenen Beitragsfinanzierung sozialer Sicherungssysteme mittelbar auf tarifpolitische Verteilungskonflikte auswirkt. Angesichts universeller Ressourcenknappheit sind auch die Lösungsperspektiven staatlicher Handlungsalternativen beschränkt. Stehen demokratisch legitimierte Akteure vor der Herausforderung, ihre Wiederwahlchancen über die Abwägung der Einkommensinteressen älterer und jüngerer Wählergruppen zu optimieren, dann legt eine Orientierung am Medianwählermodell zunächst die Vermutung nahe, dass die Versorgungsinteressen älterer Wähler die politische Programmatik dominieren werden. Allerdings zwingt die einsetzende Alterung der geburtenstarken Jahrgänge politische Akteure dazu, die Opportunitätskosten ihrer Entscheidungen zu beachten, weil die ökonomischen Konsequenzen eines ausufernden politischen Gestaltungsanspruchs für die Mehrheit der Wahlbevölkerung zeitnah und zunehmend deutlich spürbar werden. Das setzt dem Vorsorgestaat Grenzen.

Gleichwohl werden die an ihrer Wiederwahl interessierten politischen Agenten kaum auf ihren Gestaltungsanspruch verzichten, solange sozialpolitische Handlungskompetenz als relevant im Wettbewerb um die Wählergunst erachtet wird. Deshalb ist eher eine Belebung der politisch wie verfassungsrechtlich relevanten Debatte darüber zu erwarten, in welchen Fällen und in welchem Umfang der Staat berechtigt ist, individuelle Handlungsfreiheiten einzuschränken - eine Fragestellung, zu der aber ordnungsökonomische Analysen gerade aufgrund ihrer normativen Fundierung maßgeblich beitragen.

Funding Open Access funding enabled and organized by Projekt DEAL. 
Open Access Dieser Artikel wird unter der Creative Commons Namensnennung 4.0 International Lizenz veröffentlicht, welche die Nutzung, Vervielfältigung, Bearbeitung, Verbreitung und Wiedergabe in jeglichem Medium und Format erlaubt, sofern Sie den/die ursprünglichen Autor(en) und die Quelle ordnungsgemäß nennen, einen Link zur Creative Commons Lizenz beifügen und angeben, ob Änderungen vorgenommen wurden.

Die in diesem Artikel enthaltenen Bilder und sonstiges Drittmaterial unterliegen ebenfalls der genannten Creative Commons Lizenz, sofern sich aus der Abbildungslegende nichts anderes ergibt. Sofern das betreffende Material nicht unter der genannten Creative Commons Lizenz steht und die betreffende Handlung nicht nach gesetzlichen Vorschriften erlaubt ist, ist für die oben aufgeführten Weiterverwendungen des Materials die Einwilligung des jeweiligen Rechteinhabers einzuholen.

Weitere Details zur Lizenz entnehmen Sie bitte der Lizenzinformation auf http://creativecommons.org/ licenses/by/4.0/deed.de.

\section{Literatur}

Aretz, Bodo, Désirée Christofzik, und Martin Werding. 2016. Auswirkungen der Flüchtlingsmigration auf die langfristige Tragfähigkeit der öffentlichen Finanzen. SVR-Arbeitspapier Nr. 6/2016. https:// www.sachverstaendigenrat-wirtschaft.de/fileadmin/dateiablage/gutachten/jg201617/arbeitspapiere/ arbeitspapier_06_2016.pdf. Zugegriffen: 19. Okt. 2020.

Bahnsen, Lewe, Gerrit Manthei, und Bernd Raffelhüschen. 2018. Ehrbarer Staat? Die Generationenbilanz. Update 2018. Argumente zu Marktwirtschaft und Politik, Bd. 142. Berlin: Stiftung Marktwirtschaft.

Beznoska, Martin, und Jochen Pimpertz. 2016. Neue Empirie zur betrieblichen Altersvorsorge. IW-Trends 43:3-17.

Downs, Anthony. 1968. Ökonomische Theorie der Demokratie. Tübingen: Mohr Siebeck.

Ehrentraut, Oliver, Stefan Moog, und Jan Limbers. 2017. Sozialbeitragsentwicklung und Beschäftigung: Gesamtwirtschaftliche Auswirkungen steigender Beitragssätze in der Sozialversicherung und Finanzierungsalternativen. Hrsg. Prognos. https://www.prognos.com/uploads/tx_atwpubdb/20170726_ BDA_Endbericht_30052017.pdf. Zugegriffen: 19. Okt. 2020.

Eucken, Walter. 1950. Die Grundlagen der Nationalökonomie, 6. Aufl., Berlin: Springer.

Ewald, Francois. 1993. Der Vorsorgestaat. Deutsche Erstausgabe, 1. Aufl., Frankfurt: Suhrkamp.

Kochskämper, Susanna, und Jochen Pimpertz. 2015. Welche Umverteilungseffekte deckt das Solidaritätsprinzip in der GKV ab? IW-Trends 42:105-119.

Kochskämper, Susanna. 2017. Alternde Bevölkerung - Herausforderung für die Gesetzliche Krankenund für die soziale Pflegeversicherung. IW-Report Nr. 8/2017. https://www.iwkoeln.de/fileadmin/ publikationen/2017/330668/IW-Report_8_2017_Kranken_Pflegeversicherung.pdf. Zugegriffen: 23. Okt. 2020.

Kommission Verlässlicher Generationenvertrag. 2020. Empfehlungen. Bericht der Kommission, Bd. I. Bonn: BMAS.

Müller-Armack, Alfred. 1950. Soziale Irenik. Weltwirtschaftliches Archiv 64:181-203.

Pimpertz, Jochen. 2018. Mehr Differenzierung statt Einheitlichkeit. Zeitschrift für Wirtschaftspolitik 67:54-62.

Pimpertz, Jochen. 2019. Bürgerversicherung - Keine Lösung für künftige Herausforderungen in der GKV. IW Policy Paper Nr. 9/2019. https://www.iwkoeln.de/studien/iw-policy-papers/beitrag/jochenpimpertz-buergerversicherung-keine-loesung-fuer-kuenftige-herausforderungen-in-der-gkv.html. Zugegriffen: 29. Okt. 2020.

Roth, Steffen J. 2014. VWL für Einsteiger, 4. Aufl., Konstanz und München: UVK und Lucius.

Watrin, Christian. 1984. Staatsaufgaben - die ökonomische Sicht. Zeitschrift für Wirtschaftspolitik 42:131-159.

Werding, Martin, und Benjamin Läpple. 2019. Wie variabel ist der demografische Alterungsprozess? Effekte von Geburten und Zuwanderung - Folgen für die soziale Sicherung. Gütersloh: Bertelsmann Stiftung. https://doi.org/10.11586/2019015. 